\title{
Effect of Abdominal Muscle Exercises on Peak Expiratory Flow Rate in Post-Menopausal Women
}

\author{
Patil Poonam ${ }^{1}$, Sagar Javid ${ }^{2}$ \\ ${ }^{1}$ Post Graduate Student, Faculty Of Physiotherapy. Krishna Institute Of Medical Sciences Deemed University, \\ Karad, District -Satara, Maharashtra. \\ ${ }^{2}$ Associate Professor, Faculty Of Physiotherapy. Krishna Institute Of Medical Sciences Deemed University, \\ Karad, District -Satara, Maharashtra.
}

\begin{abstract}
:
Purpose of the study: To find the effect of abdominal muscle exercises on peak expiratory flow rate in postmenopausal women.

Material and Methods: 60 subjects post menopause between the age group of 45-55 years, were selected for the study.They were bounded in single group. The pre outcome measure was peak expiratory flow rate, waist hip ratio and abdominal strength .peak expiratory flow rate measured by peak expiratory flow rate device, waist hip ratio measured by inch tape and abdominal muscle strength measured by grades of abdominal muscle. The specific exercise protocol was given to the subjects which was included abdominal muscle exercises (graded abdominal muscle exercises).post treatment outcome measure were performed for peak expiratory flow rate, waist hip ratio and abdominal muscle strength. Statistical analysis was done using paired' test.

Results: In study pre-intervention peak expiratory flow rate was $290 \pm 58.251$ and post-intervention peak expiratory flow rate was $307 \pm 60.914$.Peak expiratory flow rate statistically extremely significant difference and increasing peak expiratory rate post intervention with $(p<0.0001)$ with $t=5.633$ with 59 degree of freedom.

In the study the pre intervention values of waist hip ratio was $38.683 \pm 3.000$ and post intervention waist hip ratio was 37.566 22.708.in waist hip ratio statistically extremely significant difference and reducing waist hip ratio post intervention with $(p<0.0001)$ with $t=6.996$ with 59 degree freedom.

In the study the pre intervention values of abdominal muscle strength (MMT or grades of abdominal muscle) was $1.63 \pm 0.7357$ and post intervention abdominal muscle strength was $2.5 \pm 0.7249$.In abdominal muscle strength statistically extremely significant difference and increases abdominal muscle strength with $(p<0,0001)$ with $t=11.851$ with 59 degree of freedom

Conclusion: Thus the above study it concludes that abdominal muscle exercises had significant improved clinically and statistically on peak expiratory flow rate in post-menopausal women. So this study accepts the alternate hypothesis.
\end{abstract}

Keywords: Graded Abdominal Muscle Exercises, PEFR, Menopause.

\section{Introduction}

Menopause is a natural process that occurs as a woman's ovaries stop producing eggs, and the production of hormones such as estrogen and progesterone decline. Menopause does not occur suddenly. Menopause is a sign of aging in the woman. Loss of ovarian function induces a reduction in resting metabolic rate, physical energy expenditure, fat-free mass and abdominal adipose tissue accumulation. Location of adipose tissue deposit in abdominal region plays an important role in occurrence of hyperlipidemia, diabetes, and hypertension ${ }^{[1]}$

In Normal circumstances show that at the post-menopausal age, women start gaining excessive weight around the abdominal area and there is difficulty in performing strenuous activities like stair climbing, brisk walking. Hence, there was need to find out if there is a co-relation between abdominal weight gain and lung capacity in menopausal women. ${ }^{[14]}$

Peak Expiratory Flow Rate [PEFR] ${ }^{[3]}$

The peak expiratory flow (PEF), also called peak expiratory flow rate (PEFR) is a person's maximum speed of expiration, as measured with a peak flow meter, a small, hand-held device used to monitor a person's ability to breathe out air. It measures the airflow through the bronchi and thus the degree of obstruction in the airways

Graded abdominal muscle exercises include ${ }^{[4,15,29]}$

Upper abdominal exercises-

Starting position-The subjects were instructed to lie in supine with the hips at 45 degree and knees at 90 degree and hand at sides. In all these activities subjects were instructed to keep the low back flat. 
Grade1-subjects were asked to perform the curl ups by contracting abdominal muscles and then lifting the head off table with flexed knees.

Grade 2-the progression was made by lifting the shoulders until the top of scapulae lift from table, keeping the arms extended towards knees.

Grade 3- The next progression was done by lifting the shoulders until the scapulae clear table, keeping the arms horizontal.

Grade 4- The subjects were asked to progresses further by keeping the arms crossed over chest, until scapulae clear table.

Grade 5- The subjects were asked to progress the difficulty of the curl ups by having the subject change the arm position from horizontal and then to behind the neck, until scapulae clear table.

\section{Participants}

60 women diagnosed with menopause of age 45-55 years in Krishna hospital and Peth area were been participated in the study. They were bounded in single group. The pre outcome measure was peak expiratory flow rate, waist hip ratio and abdominal strength .peak expiratory flow ate measured by peak expiratory flow rate device, waist hip ratio measured by inch tape and abdominal muscle strength measured by grades of abdominal muscle. The specific exercise protocol was given to the subjects which was included abdominal muscle exercises (graded abdominal muscle exercises).

\section{Outcome measures:}

1. Peak expiratory flow rate -:The peak expiratory flow (PEF), also called peak expiratory flow rate (PEFR) is a person's maximum speed of expiration, as measured with a peak flow meter, a small, hand-held device used to monitor a person's ability to breathe out air. It measures the airflow through the bronchi and thus the degree of obstruction in the airways. ${ }^{[3]}$

2. Waist Hip ratio-: Waist-hip ratio or waist-to-hip ratio (WHR) is the ratio of the circumference of the waist to that of the hips. This is calculated as waist measurement divided by hip measurement (W $\div \mathrm{H}$ ). For example, a person with a 25" $(64 \mathrm{~cm})$ waist and 38" $(97 \mathrm{~cm})$ hips has a waist-hip ratio of about 0.66.The WHR has been used as an indicator or measure of health, and the risk of developing serious health conditions. WHR correlates with fertility (with different optimal values for males and females).

3. MMT for abdominal muscle ${ }^{[5]}$

Graded abdominal muscle exercises include

Upper abdominal exercises-

Starting position-The subjects were instructed to lie in supine with the hips at 45 degree and knees at 90 degree and hand at sides. In all these activities subjects were instructed to keep the low back flat.

Grade1-subjects were asked to perform the curl ups by contracting abdominal muscles and then lifting the head off table with flexed knees.

Grade 2-the progression was made by lifting the shoulders until the top of scapulae lift from table, keeping the arms extended towards knees.

Grade 3- The next progression was done by lifting the shoulders until the scapulae clear table, keeping the arms horizontal.

Grade 4- The subjects were asked to progresses further by keeping the arms crossed over chest, until scapulae clear table.

Grade 5- The subjects were asked to progress the difficulty of the curl ups by having the subject change the arm position from horizontal and then to behind the neck, until scapulae clear table.

\section{Procedure:}

A subject who was fulfilling the inclusion and exclusion criteria was included. Informed consent form was taken from each of the subjects prior to treatment. An instruction was given to the subjects about techniques performed. Prior to the treatment abdominal girth was checked by inch tape method.Prior to the treatment MMT of abdominal was checked .Prior intervention peak expiratory flow rate was checked.Peak expiratory flow rate checked by peak expiratory flow rate device.In this study, graded abdominal muscle exercise protocol was prescribed to the subjects.A set protocol of graded abdominal muscle exercise was demonstrated and taught to the subject. 3 set of each exercises was given to the subject for 6 times per week for 20 repetitions for duration 1 months ${ }^{[7]}$.The effect of graded abdominal exercises was noticed and measured using the peak expiratory flow rate and abdominal girth measurement checked with using inch tape. 


\section{Methodology}

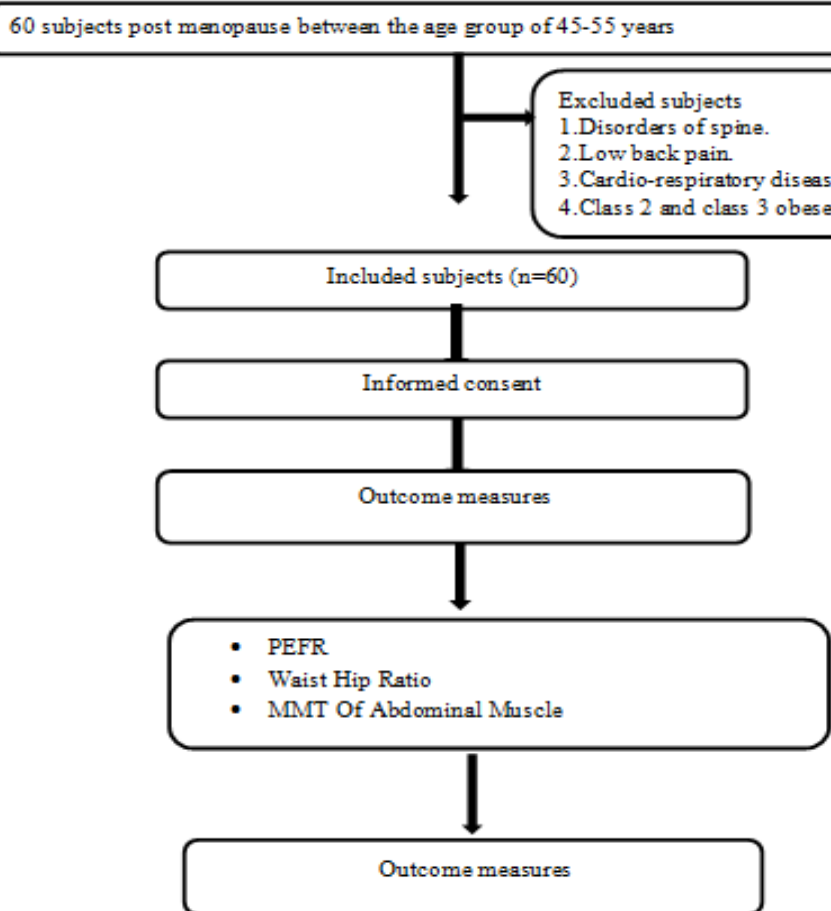

Statistical Analysis

Statistical analysis was done by using INSTAT software, version 3.10. Within group analysis was done using paired " $\mathrm{t}$ " test.

\section{1) Peak Expiratory Flow Rate:}

\section{Results}

The pre intervention peak expiratory flow rate vale were $290 \pm 58.251$, whereas post intervention the value was $307.33 \pm 60.914$.the post intervention change in peak expiratory flow rate values showed statistically extremely significant. The "P" value is $<0.0001$ considered extremely significant .This was done using paired" test. ' $\mathrm{t}$ ' value is $\mathrm{t}=5.633$ with 59 degree of freedom.

\begin{tabular}{|l|l|l|}
\hline Pefr & Pre Pefr & Post Pefr \\
\hline Mean & 290 & 307.33 \\
\hline SD & 58.251 & 60.914 \\
\hline
\end{tabular}

Table 1:Mean and Standard deviationof PEFR

2) Waist Hip Ratio:

The pre interventional waist hip ratio value was $38.683 \pm 3.000$ whereas post interventional the value was $37.566 \pm 2.7088$.the post interventional change in waist hip ratio value showed statistically extremely significant. The "P" value is $<0.0001$ considered extremely significant this was doing using paired" t" test the " $\mathrm{t}$ " value is $\mathrm{t}=6.996$ with 59 degree freedom.

\begin{tabular}{|l|l|l|}
\hline WHR & PRE WHR & POST WHR \\
\hline MEAN & 38.683 & 37.566 \\
\hline SD & 3.000 & 2.7088 \\
\hline
\end{tabular}

3) Manual Muscle Testing :

Table 2: Mean and SD of waist hip ratio

The pre interventional MMT value was $1.63 \pm 0.7357$ whereas post interventional the value was $2.5 \pm 0.7249$.the post interventional change in value showed statistically extremely significant. The "P" value is $<0.0001$ considered extremely significant .this was doing using paired" $t$ " test .the " $t$ " value is $t=11.851$ with 59 degree freedom. 


\begin{tabular}{|l|l|l|}
\hline MMT & PRE MMT & POST MMT \\
\hline MEAN & 1.63 & 2.5 \\
\hline SD & 0.7357 & 0.7249 \\
\hline
\end{tabular}

Table3: Mean and SD of MMT

\section{Discussion}

Menopause is a natural process that occurs as a woman's ovaries stop producing eggs, and the production of hormones such as estrogen and progesterone decline. Menopause does not occur suddenly. Menopause is a sign of aging in the woman. Loss of ovarian function induces a reduction in resting metabolic rate, physical energy expenditure, fat-free mass and abdominal adipose tissue accumulation. Location of adipose tissue deposit in abdominal region plays an important role in occurrence of hyperlipidemia, diabetes, and hypertension. In Normal circumstances show that at the post-menopausal age, women start gaining excessive weight around the abdominal area and there is difficulty in performing strenuous activities like stair climbing, brisk walking. Hence, there was need to find out if there is a co-relation between abdominal weight gain and lung capacity in menopausal women ${ }^{[14]}$. Relation between abdominal fat and menopause-Menopause is a sign of aging in the woman. Loss of ovarian function induces a reduction in resting metabolic rate, physical energy expenditure, fat-free mass and abdominal adipose tissue accumulation.60 women diagnosed with menopause of age 45-55 years in Krishna hospital and Peth area were been participated in the study. They were bounded in single group. The pre outcome measure was peak expiratory flow rate, waist hip ratio and abdominal strength .peak expiratory flow ate measured by peak expiratory flow rate device, waist hip ratio measured by inch tape and abdominal muscle strength measured by grades of abdominal muscle. The specific exercise protocol was given to the subjects which was included abdominal muscle exercises (graded abdominal muscle exercises).post treatment outcome measure were performed for peak expiratory flow rate, waist hip ratio and abdominal muscle strength. Statistical analysis was done using paired't' test.

In study pre-intervention peak expiratory flow rate was $290 \pm 58.251$ and post-intervention peak expiratory flow rate was $307 \pm 60.914$.in peak expiratory flow rate statistically extremely significant difference and increasing peak expiratory rate post intervention with $(\mathrm{p}<0.0001)$ with $\mathrm{t}=5.633$ with 59 degree of freedom. In the study the pre intervention values of waist hip ratio was $38.683 \pm 3.000$ and post intervention waist hip ratio was 37.566 \pm 2.708 .in waist hip ratio statistically extremely significant difference and reducing waist hip ratio post intervention with $(\mathrm{p}<0.0001)$ with $\mathrm{t}=6.996$ with 59 degree freedom.

In the study the pre intervention values of abdominal muscle strength (MMT or grades of abdominal muscle) was $1.63 \pm 0.7357$ and post intervention abdominal muscle strength was $2.5 \pm 0.7249$.In abdominal muscle strength statistically extremely significant difference and increases abdominal muscle strength with $(\mathrm{p}<0,0001)$ with $\mathrm{t}=11.851$ with 59 degree of freedom.

\section{Conclusion}

Thus the above study it concludes that abdominal muscle exercises had significant improved clinically and statistically on peak expiratory flow rate in post-menopausal women. So this study accepts the alternate hypothesis

\section{Conflicts Of Interest}

Nil

\section{Source Of Funding}

Krishna Institute Of Medical Sciences Deemed University, Karad.

Ethical Clearance

Study approved by Institutional Ethics Committee of Krishna Institute of Medical Sciences, Karad.

\section{Bibliography}

[1]. Margaret Polden And Jill MantleText Book Of “Physiotherapy In Obstetrics And Gynecology"first edition ,page no 287.

[2]. K Sembulingam And PremaSembulingam Text Book Of "Essential Of Medical Physiology " $5^{\text {th }}$ Edition ,Page No 645.

[3]. Donna FrownfelterAnd Elizabeth Dean. Text Book Of "Cardiovascular And Pulmonary Physical Therapy" Evidence To Practice. $5^{\text {th }}$ edition page no 137

[4]. Carolyn Kisner And Lynn Allen Colby Text Book Of "Therapeutic Exercises Foundation And Techniques.4 $4^{\text {th }}$ Edition, Page no 663

[5]. "Vispute, Sachin S; Smith, John D; Lecheminant, James D; Hurley, Kimberly S . "The Effect Of Abdominal Exercise On Abdominal Fat".

[6]. Ana C.M Renno, RenotaN.Granita, PatricaDriusso, Dirceu Costa ,Jorge OishiEffect Of An Exercise Program On Respiratory Function, Thoracic Kyphosis, Tolerance To Exercises’ And Quality Of Life In Women With Osteoporosis.

[7]. MaftabMoacame, SamanhFarahatiEffectOf Aerobic Training On Pulmonary Functions On Post-Menopausal Women.

[8]. Ogwamike O.O ArowojoluAo And Sanya A.OT The Effects Of A12-Week Endurance Exercise Program On Adiposity And Flexibility Of Nigerian Perimenopausal And Postmenopausal Women.

[9]. Melinda L. Irwin, YutakaYasui, Cornelia M. Ulrich, Deborah Bowen, Rebecca E. Rudolph, Etol The Effect Of Exercise On Total And Intra-Abdominal Body Fat In Postmenopausal Women. 
[10]. Chaitra B, PandurangNarahare, NagarajaPuranik, Vijay MaitriThe Effect Of Aerobic Exercises Training On Pulmonary Function Test .

[11]. M.L. Maltais, J. Desroches, I.J. Dionne The Change In Muscle Mass After Menopause Emmanuel Gomes Ciolac Julia Maria D'AndreaGreve The Exercise-Induced Improvements In CardioRespiratory Fitness And Heart Rate Response To Exercise Are Impaired In Overweight And Obese Postmenopausal Women.

[12]. Jack Wilmoor, David Costil The Text Book Of "Physiology And Exercises Sports Menopause linked with reduced lung function, more lung symptomsNEW YORK (Reuters Health) - There appears to be an association between menopause and a reduced lung function plus more respiratory symptoms, especially among lean women, European researchers report.

[13]. Florence Peterson Kendall, Elizabeth Kendall, Patricia GeiseProvanceThe text book of muscles testing and function fourth edition page no 133.

[14]. Frederiksberg C, Denmark. "The Physical Activity And Weight Gain And Fat Distribution Changes With Menopause".

[15]. Green JS1, Stanforth PR, Rankinen T, Leon AS, Rao Dc Dc, Skinner JS, Bouchard C, Wilmore JH.” The effects of exercise training on abdominal visceral fat, body composition, and indicators of the metabolic syndrome in postmenopausal women

[16]. De Lorenzo A.a • Petrone-De Luca P.a • SassoG.b • CarbonelliM.c • Rossi P.d • BrancatiA.a "The effect of Weight Loss on Body Composition and Pulmonary Function"

[17]. Pelosi P1, Quintel M, Malbrain ML." The effect of intra-abdominal pressure on respiratory mechanics"

[18]. Colombel A1, Charbonnel B.1997:"The Weight gain and cardiovascular risk factors in the post-menopausal women'"

[19]. Heymsfield SB1, Gallagher D, Poehlman ET, Wolper C, Nonas K, Nelson D, Wang ZM1994: "The effect of Menopausal changes in body composition and energy expenditure'"

[20]. Toth MJ1, Tchernof A, Sites CK, Poehlman ET.2009:"The Menopause-related changes in body fat distribution".

[21]. Tchernof A1, Poehlman ET 1998: "The Effects of the menopause transition on body fatness and body fat distribution"

[22]. Green JS1, Stanforth PR, Rankinen T, Leon AS, Rao Dc Dc, Skinner JS, Bouchard C, Wilmore JH1997" The effects of exercise training on abdominal visceral fat, body composition, and indicators of the metabolic syndrome in postmenopausal women"

[23]. P.H. Quanjer, M.D. Lebowitz, I. Gregg, M.R. Miller, O.F. Pedersen1997'The Peak expiratory flow -conclusions and recommendations of a Working Party of the European Respiratory Society ".

[24]. V Brusasco 2003:"The Usefulness of peak expiratory flow measurements"

[25]. IshidHWatanabs 2014"The Maximum expiration activates the abdominal muscles during side bridge exercise"

[26]. P.Kendall 1993 florence.

[27]. David J. Magee, "Orthopedic Physical Assessment" $4^{\text {th }}$ Edition. 